\title{
An Improved High Performance Liquid Chromatographic Method for Identification and Quantization of Polyamines as Benzoylated Derivatives
}

\author{
Rajat Sethi $^{1 \#}$, Sai Raghuveer Chava ${ }^{2}$, Sajid Bashir ${ }^{2,3 \S}$, Mauro E. Castro ${ }^{2 \#}$ \\ ${ }^{1}$ Cardiovascular Research and Development Laboratory, Department of Pharmaceutical Sciences, \\ Rangel College of Pharmacy, Texas A\&M Health Science Center, Kingsville, USA \\ ${ }^{2}$ Department of Chemistry, Texas A\&M University-Kingsville, Kingsville, USA \\ ${ }^{3}$ Chemical Biology Research Group (CBRG), 700 University Blvd, Kingsville, USA

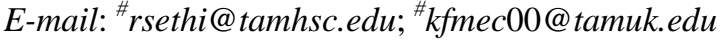 \\ Received April 25, 2011; revised May 23, 2011; accepted June 23, 2011
}

\begin{abstract}
A simple reversed phase high performance liquid chromatography (RP-HPLC) method was developed for the determination of putrescine, cadaverine and spermidine (a class of polyamines) in their benzoylated form from external known standards. In the optimization procedure, a number of parameters were examined: 1) Solvent used in the extraction of standard polyamines (diethyl ether versus chloroform); 2) Solvent used in the elution of the polyamine (methanol versus acetonitrile); 3) Mode of derivatization and extraction step(s) (derivatization and extraction performed together versus derivatization and extraction performed separately); and 4) Other instrumental parameters (such as UV detection wavelength, gradient profiles). The advantages of our method, relative to the standard Morgan method are: a) decreased chromatographic runtime, b) ease of preparation with good resolution, sensitivity, and reproducibility using a standard RP-HPLC method.
\end{abstract}

Keywords: Polyamine, RP-HPLC, Standardization, Benzoylation

\section{Introduction}

Cardiovascular diseases (CVD, *) are a major cause for death of people in North America and have been attributed to a number of epidemic factors [1]. One potential mechanism for the increased incidence of CVDs affecting 81million Americans is alteration in polyamine content in the heart [2]. Other risk factors associated with CVD are high blood pressure, poor diet and obesity. Clinical studies have demonstrated that intake of polyamines from external sources can minimize the incidence of CVD [3]. Polyamines are a class of polycationic compounds, which are ubiquitous in many tissues of mammals and other organisms. The three most common members of the polyamine

*This work was supported by grants from United States Environmental Protection Agency Grant (USEPA Grant \# IT-83404401-0), Texas A\&M Health Science Research Development Grant (Act\# 134403-35402) and funds from TAMHSC Research Startup (Ac\$\# 13100-35488), Robert A. Welch Foundation (Departmental Grant AC006) and National Science Foundation (NSF) Division of Undergraduate Education (DUE) Program (9987332) for financial support.

${ }^{\S}$ Dedicated to: Rebecca West, Larry Kuchta and Prabin Maharjan. family are: cadaverine, putrescine and spermidine which are low molecular weight aliphatic compounds containing nitrogen, are necessary for cell growth and differentiation in living cells [4-6]. Furthermore, polyamines consist between three to five methylene groups between the terminal nitrogen atoms. Early diagnosis and detection would allow treatment and decrease CVD attributed mortality and a number of clinical and analytical approaches have been utilized towards this end. Previous studies using urine sample of cancer patients allowed determination of polyamine to be conducted using a chromatographic approach, where correlation was observed between patients with and without disease and amount of polyamine [7]. Endogenous cadaverine is synthesized using lysine as the starting material via lysine decarboxylase (scheme shown in Figure 1). Similarly, putrescine is synthesized through a decarboxylation step using ornithine and spermidine was formed by the action of spermidine synthase, which attaches putrescine to an aminopropyl group from decarboxylated S-adenosylmethionine [8-11]. The synthesized polyamines are used by 


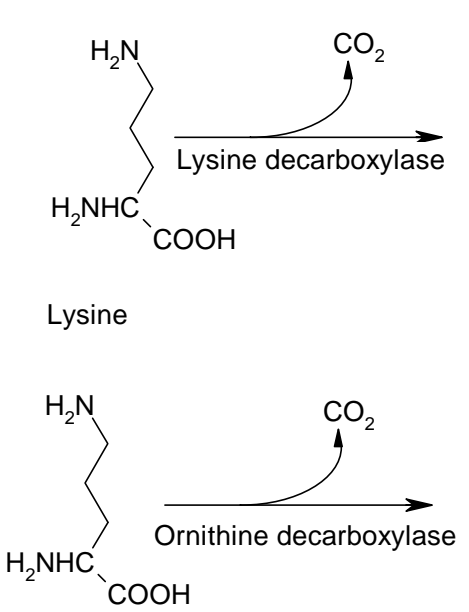

Ornithine

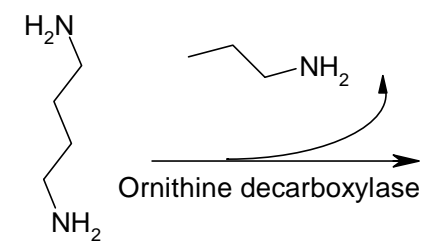

Putrescine

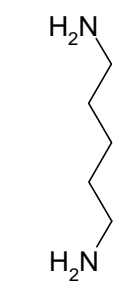

Cadaverine

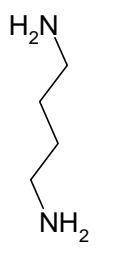

Putrescine

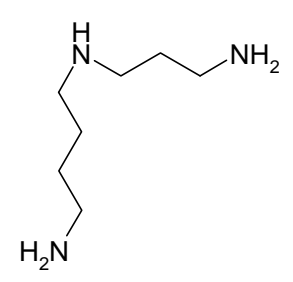

Spermidine

Figure 1. Synthesis of polyamines (Cadaverine, Putrescine and Spermidine).

the organisms for a number of biological processes, however if the levels are above or below certain biological tolerances, a disease state can result, for example if red blood cells (RBC) contains high levels of polyamines, certain tumors can result [12-15] which can be monitored through analysis of biological matrices such as urine or plasma $[16,17]$.

As previously stated a number of analytical methods can be used to monitor changes in polyamine content from biological tissue, the most common being reverse phase high performance liquid chromatography (RP-HPLC), which allows for separation, detection and quantification of polyamines (namely putrescine, cadaverine and spermidine).

The current analytical aim was to characterize polyamines using an enhanced methods, where 'enhanced' refers to a finalized procedure yielding greater analytical speed ('run time'), greater peak sensitivity (measured as signal-to-noise ratio) and better precision. In our approach, polyamines were benzoylated using benzyl chloride with 1,6-diamino hexane as the internal standard [18]. In comparison, with methods described in the literature polyamine were identified and separated in 40 minutes using water and methanol as solvent system using wavelength of 254 $\mathrm{nm}$ [19]. An alternative HPLC method required 20 minutes

for detection and isolation of polyamines using wavelength of $254 \mathrm{~nm}$ [20]. Both methods utilized (O-Phthalaldehyde) OPA type columns, lastly, a method described by Smith and Davis, required 30 minutes for identification and separation of polyamines using wavelength of $254 \mathrm{~nm}$ [21].

Since the above methods required a number of complex procedures, an attempt was made to simplify the procedure described herein. We report a revised procedure, in which the sample is derivatized, eluted and characterized using a C-18 column in the benzoylated form.

\section{Experimental Setup}

Unless otherwise stated, all chemicals were reagent grade, with ultrapure water *.

\subsection{Materials and Methods}

All Chemicals unless otherwise specified were obtained from VWR International (West Chester, PA) or SigmaAldrich (St Louis, MO). The solvents were analytical grade and were filtered using a 0.2-micron filter. Ultrapure water (Milli-Q, $18 \mathrm{M}$ ) was used to makeup any buffers or binary solvents and used to dissolve the polyamine standards

\subsection{Materials}

Benzoyl chloride, 1,6-diaminohexane were purchased from Alfa Aesar (Ward Hill, MA), Cadaverine dihydrochloride was purchased from Pfaltz Waterbury, CT) and Bauer, Inc (Waterbury, CT), Putrescine dihydrochloride was purchased from Spectrum chemicals Mfg Corp (Gardena, CA) and Spermidine trihydrochloride was purchased from Calbiochem (San Diego, CA). HPLC grade acetonitrile was obtained from Fisher chemicals (Tustin, CA). HPLC grade methanol was obtained from J.T Baker (Phillipsburg, NJ) and all other chemicals and reagents were obtained from Sigma-Aldrich (St. Louis, MO).

\subsection{Equipment}

Waters HPLC (Milford, MA) was equipped with automated sampler was used to carry out the separation of benzoylated polyamines. Provided with binary HPLC pump, $20 \mu \mathrm{L}$ Rhenodyne loop injector (Cotati, CA) and $5 \mu \mathrm{m}$ particle size harmony C-18 reverse-phase column from ES Industries (VWR International, Bridgeport, NJ). Benzoylated polyamines were detected using UV Beckman detector at $229 \mathrm{~nm}$. The output from this detector was quantified and integrated using Breeze software. All experiments were performed at room temperature. The prepared standards were stored at $-20^{\circ} \mathrm{C}$ for up to three 
months, $-70^{\circ} \mathrm{C}$ for one year use.

\subsection{Benzoylation Procedure}

To a sample (of $1 \mathrm{ml}$ ) containing 50 mole amines 1 molar equivalent (5 $\mathrm{\mu l}, \mathrm{HCl})$ ( 1 molar equivalent) was added. The sample was shaken briefly using a vortex mixer and to this, the basic internal standard was added. This consisted of $5 \mu$ l of 1,6-diamino hexane (internal standard) and $995 \mu$ l of $2 \mathrm{~N}$ sodium hydroxide (base). The basic polyamine was derivatized using ( $5 \mathrm{ml}$ ) which benzoyl chloride was added to the solvent mixture and was mixed for about 20 minutes at constant temperature. The derivatized polyamine containing both organic and aqueous compounds was separated using centrifugation ( $\times 15,000 \mathrm{~g}, 15 \mathrm{~min}$ ). After centrifugation, the upper organic phase was removed and used in the extraction of the polyamines. The organic layer was then extracted twice with diethyl ether $(2 \mathrm{ml} \times 2)$ and the organic layers combined. The extracted layers were dried in a stream of nitrogen. The remaining residue was dissolved in acetonitrile $(100 \mu \mathrm{l})$. The extracted polyamines were approximately of $50 \mu$ moles and diluted to $0.5 \mu$ moles in 1 $\mathrm{mL} .20 \mu \mathrm{l}$ of the polyamines was characterized using RP-HPLC at wavelength of $229 \mathrm{~nm}$ [22,23].

\subsection{HPLC Analysis}

$20 \mu \mathrm{l}$ (< 50 umoles) was injected and separated using the C-18 (harmony, VWR International) column, initially eluted using a gradient elution profile. The elution profile began with $70 \%$ of solvent A (acetonitrile) to $100 \% \mathrm{~A}$ over 2 minutes, then to $100 \%$ of A over 13 minutes and finally to $100 \%$ of $\mathrm{A}$ to $70 \%$ of A over 2 minutes. The flow rate was $1 \mathrm{~mL} / \mathrm{min}$ and the total run time with method 1 was $15 \mathrm{~min}$ [24]. The polyamines were extracted with either diethyl ether as described previously or with chloroform $(2 \mathrm{ml} \times 2)$ with everything else remaining the same and both characterized using the same chromatographic method.

\section{Results}

The relative merits of the extraction approach will presented and for the preferred extraction method the analytical accuracy, precision, signal-to-noise is presented followed by a comparison of the characterization of the polyamines standards with a comparison with current literature methods concluding with benefits of our approach (see Tables 1, 2).

Linear regression was employed which was mainly deals with summarizing the significance of the obtained data with two or more variables. To fit a curve to the given data, a combination of statistical techniques along with regression analysis for determination of valid data points within a data were used.

The regression analysis was used for prediction and forecasting. The regression analysis fit was linearlized as an equation " $\mathrm{Y}=\mathrm{m} \mathrm{X}+\mathrm{C}$ " where " $\mathrm{m}$ " is the slope of the line and " $C$ " the $y$-intercept which are constants [25,26]. Table 2 indicates the linear regression parameters of a set of individual standards. The polyamines standards were analyzed using the new HPLC method which gave more accurate results (summarized in Table 1).

Table 1 gives information regarding various different retention characteristics of three polyamines found in biological tissues and also in body fluids. Good separation of these polyamines was achieved (separation of polyamine mixture is shown in Figure 2, one example of improvement in peak intensity with methanol or acetonitrile extracted with diethyl ether, or chloroform is shown in Figure $\mathbf{3}$ and $\mathbf{4}$ respectively), values with excellent precision. The improvements in peak intensity were observed with all of the polyamines evaluated (raw chromatograph for the other polyamines are shown similarly extracted with diethyl ether is also shown in Figures 5-8). Two possible sources of instrumental artifacts could affect the measured values. These can be attributed to the ageing of the column may result in increased line width of the peaks. If there was an obstruction in the column, it will only result in delayed values with an increase in pressure. But, as the same resolution was measured and the samples were centrifuged and filtered, the two aforementioned instrumental artifacts were not observed.

\subsection{Standard Curves}

The correlation coefficients were more than 0.997 for the concentrations which were investigated. The linearity for all the polyamines were exhibited using absolute amount to that of area under the curves (Table 2). The concentrations which were investigated were $0.5,0.25,0.125$, 0.0625, 0.0312, 0.0156, 0.0078, 0.0036 micromoles for Putrescine (Put), Cadaverine (Cad) and Spermidine (Spd) [27].

\subsection{Recovery and Precision}

500 pmol of each compound was combined and recovered, from which recovery percentage and quantification

Table 1. Different polyamines with retention times.

\begin{tabular}{ccc}
\hline Compound & Retention time (min) & Variance (\%) \\
\hline Putrescine & 4.10 & 0.33 \\
Cadaverine & 5.41 & 0.28 \\
Spermidine & 6.24 & 0.14 \\
\hline
\end{tabular}


Table 2. Linear Regression Parameters of Individual Standards Set.

\begin{tabular}{cccc}
\hline Compound & Slope & Intercept & Correlation Coefficient $\left(\mathrm{R}_{2}\right)$ \\
\hline Putrescine & $3.00 \mathrm{E}+06$ & 8999.3 & 0.999 \\
Cadaverine & $2.00 \mathrm{E}+07$ & 216365 & 0.997 \\
Spermidine & $1.00 \mathrm{E}+07$ & 212088 & 0.998 \\
\hline
\end{tabular}

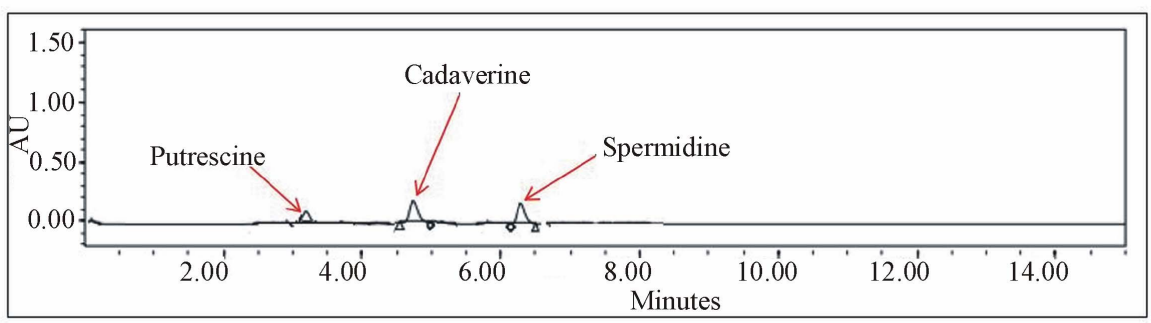

Figure 2. Chromatogram of 3 standards extracted using diethyl ether and separated with acetonitrile.
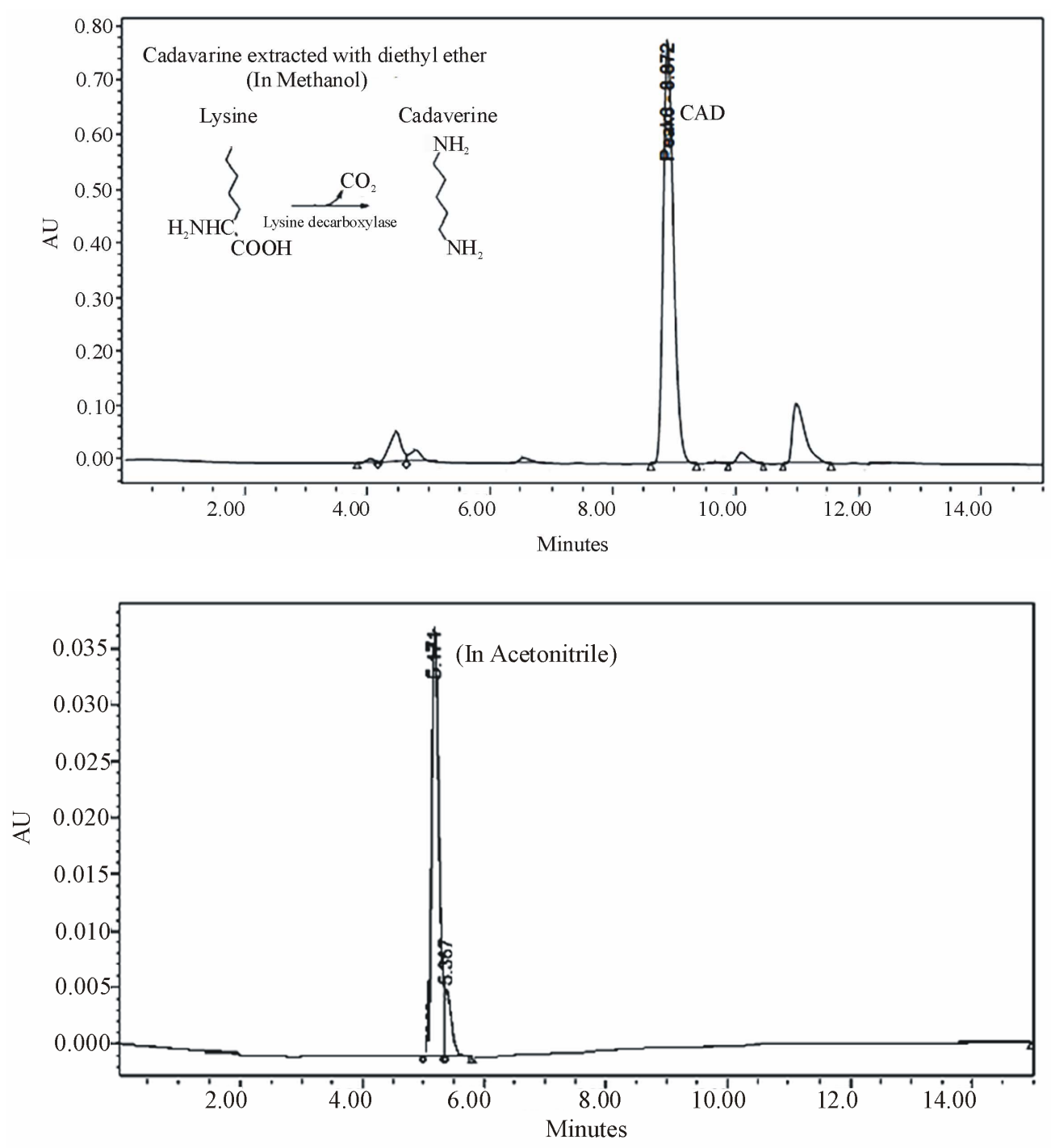

Figure 3. Standard chromatogram's of cadaverine extracted with diethyl ether but separated with methanol or acetonitrile. 

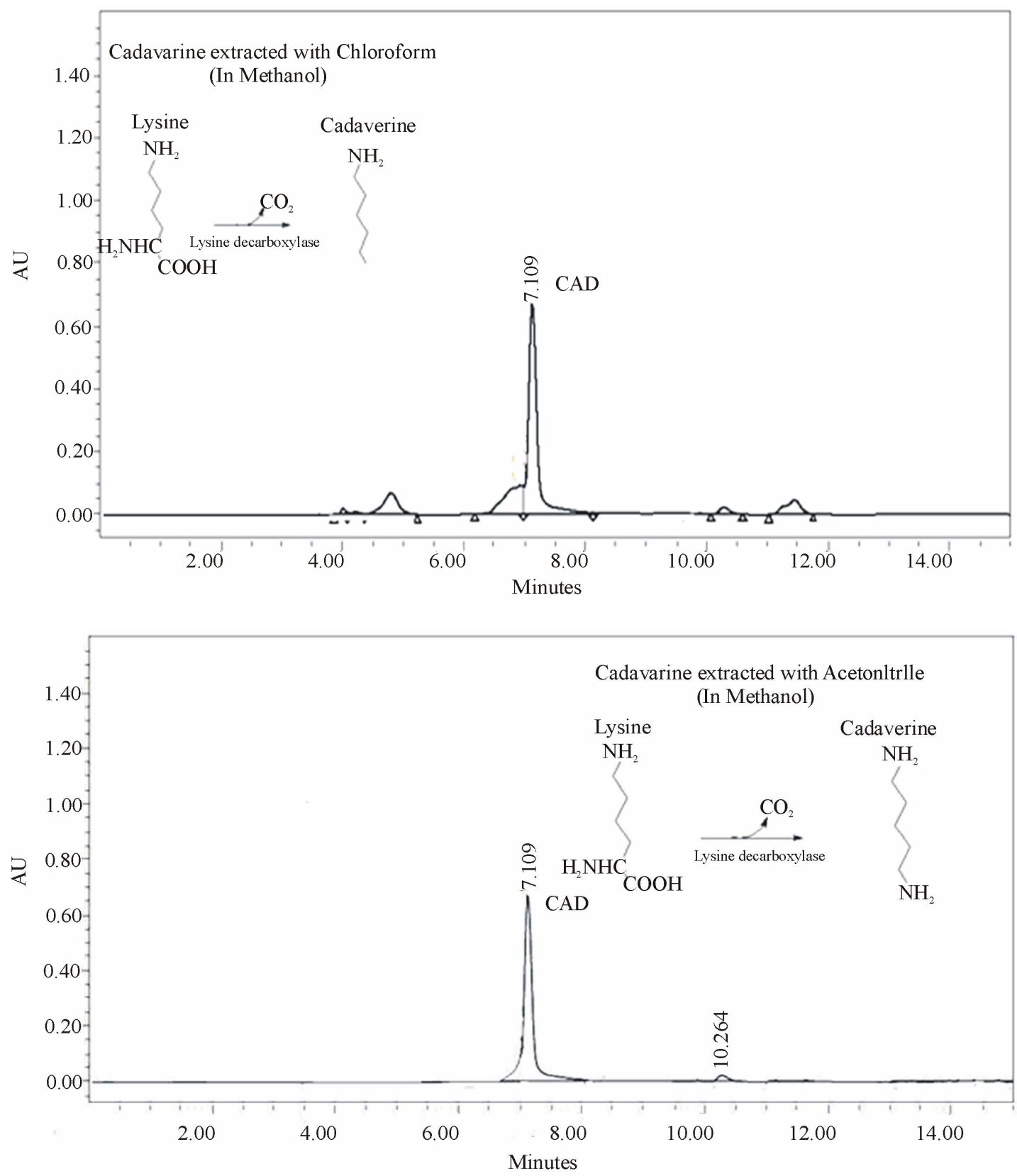

Figure 4. Standard chromatogram's of cadaverine extracted with chloroform but separated with methanol or acetonitrile.

using:

$$
\mathrm{R}=\mathrm{X} 2 / \mathrm{X} 1 \times 100
$$

where $\mathrm{X} 1=$ Weigh value and $\mathrm{X} 2$ = Calculated value.

This procedure was repeated 3 times a day for 3 consecutive days and analyzed for variance and precision. The variance was between $88 \%$ and 125\% (Table 3) with $99 \%$ precision.

The interday coefficient of variation was calculated from results of three consecutive days for mixture containing 500 pmoles of each polyamine. Also the intraday variation coefficient was calculated from 3 determina tions of mixture which contained 500 pmoles of each individual compound [28,29].

The detection limit considered to be absolute amount which generated a signal 5 times the baseline was observed. The lowest detection limit for putrescine, cadaverine and spermidine was 5 pmol with diaminohexane as internal standard with excellent differentiation and retention time.

Cadaverine was extracted with diethyl ether or chloroform and seperated with methanol or acetonitrile. Cadaverine extracted with diethyl ether and seperated 
R. SETHI $E T \quad A L$.

461

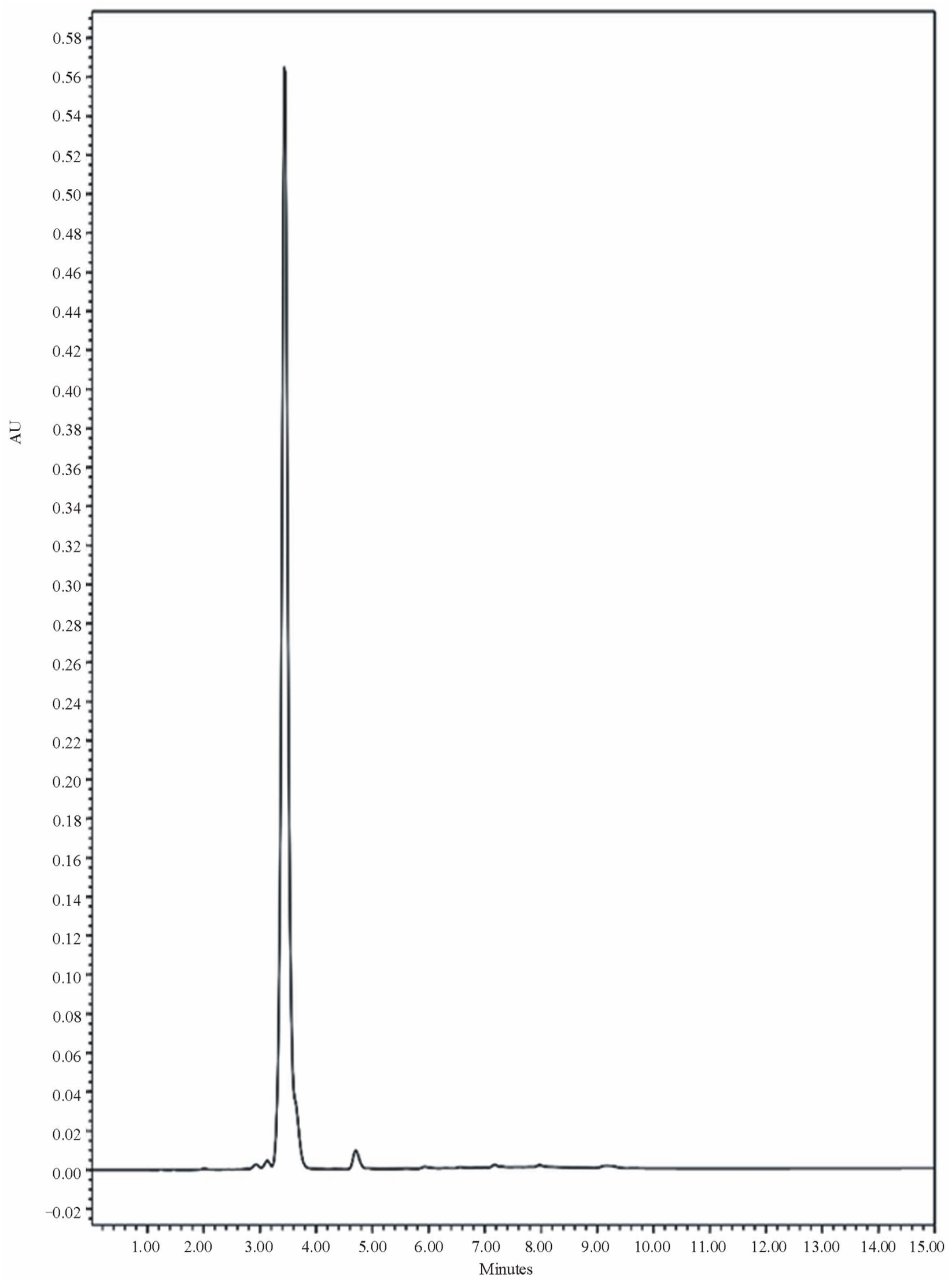

Copyright (C) 2011 SciRes.

AJAC 


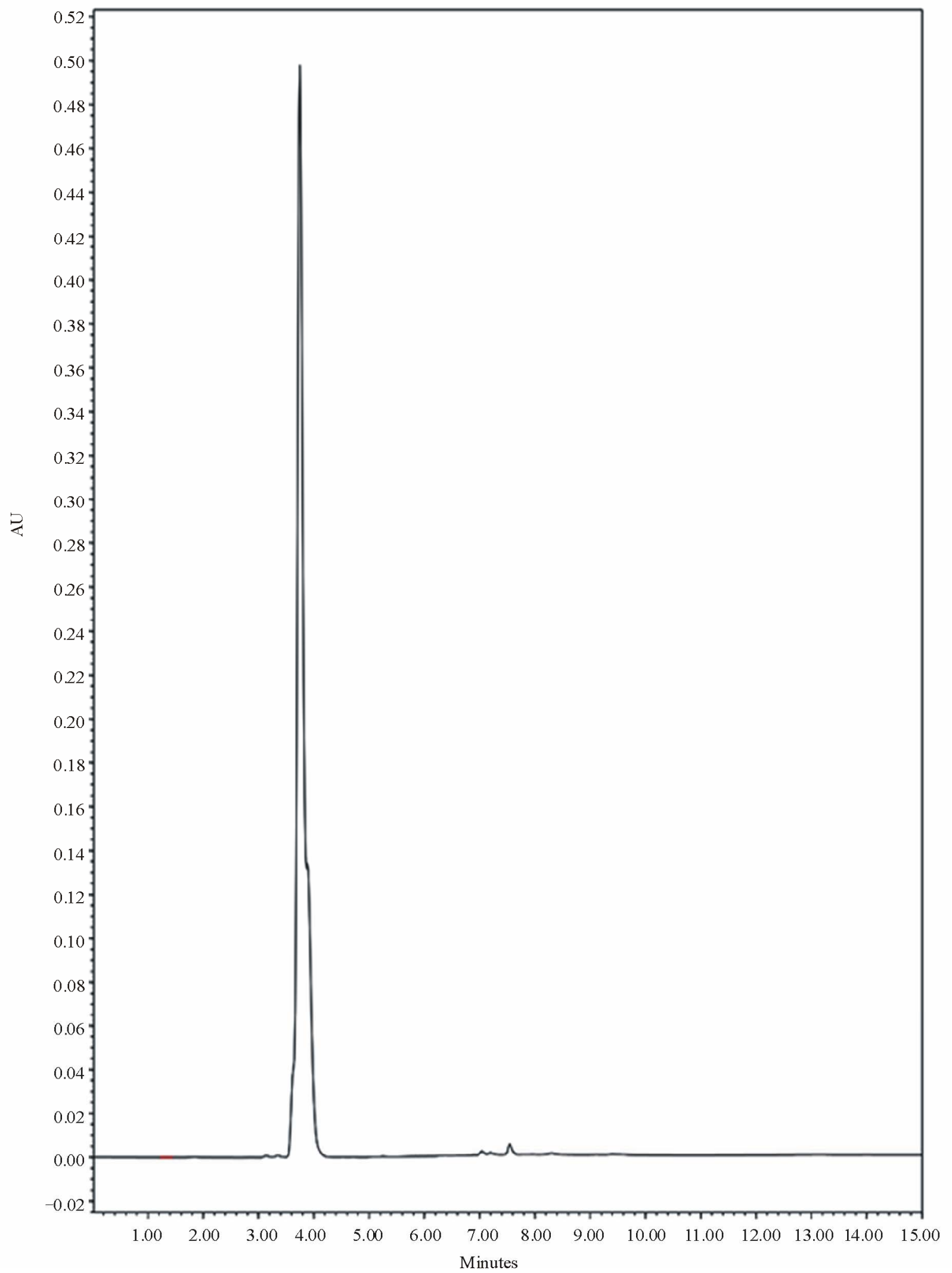

Figure 5. Standard chromatogram's of putrescence extracted with diethyl ether but separated with methanol or acetonitrile. 


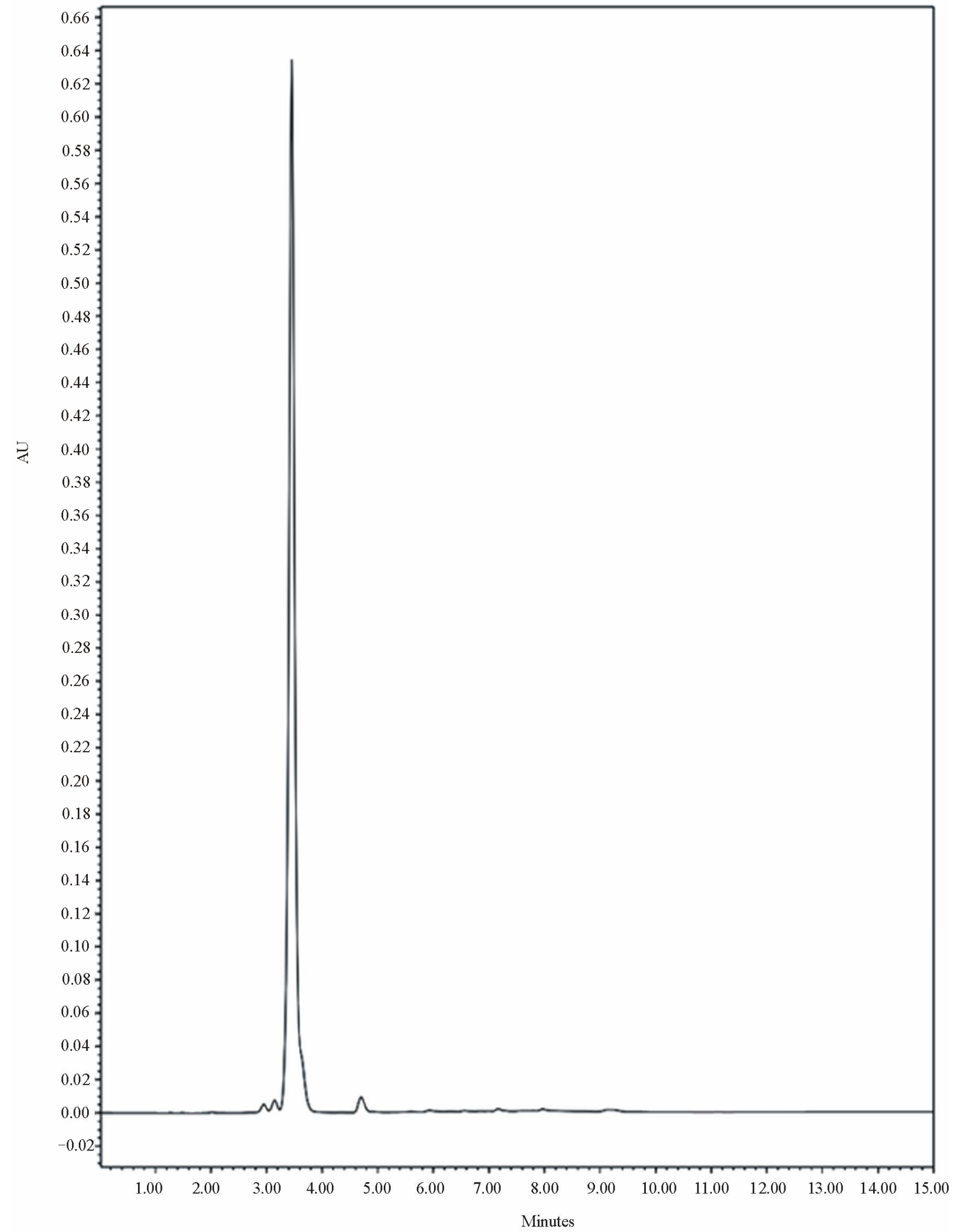




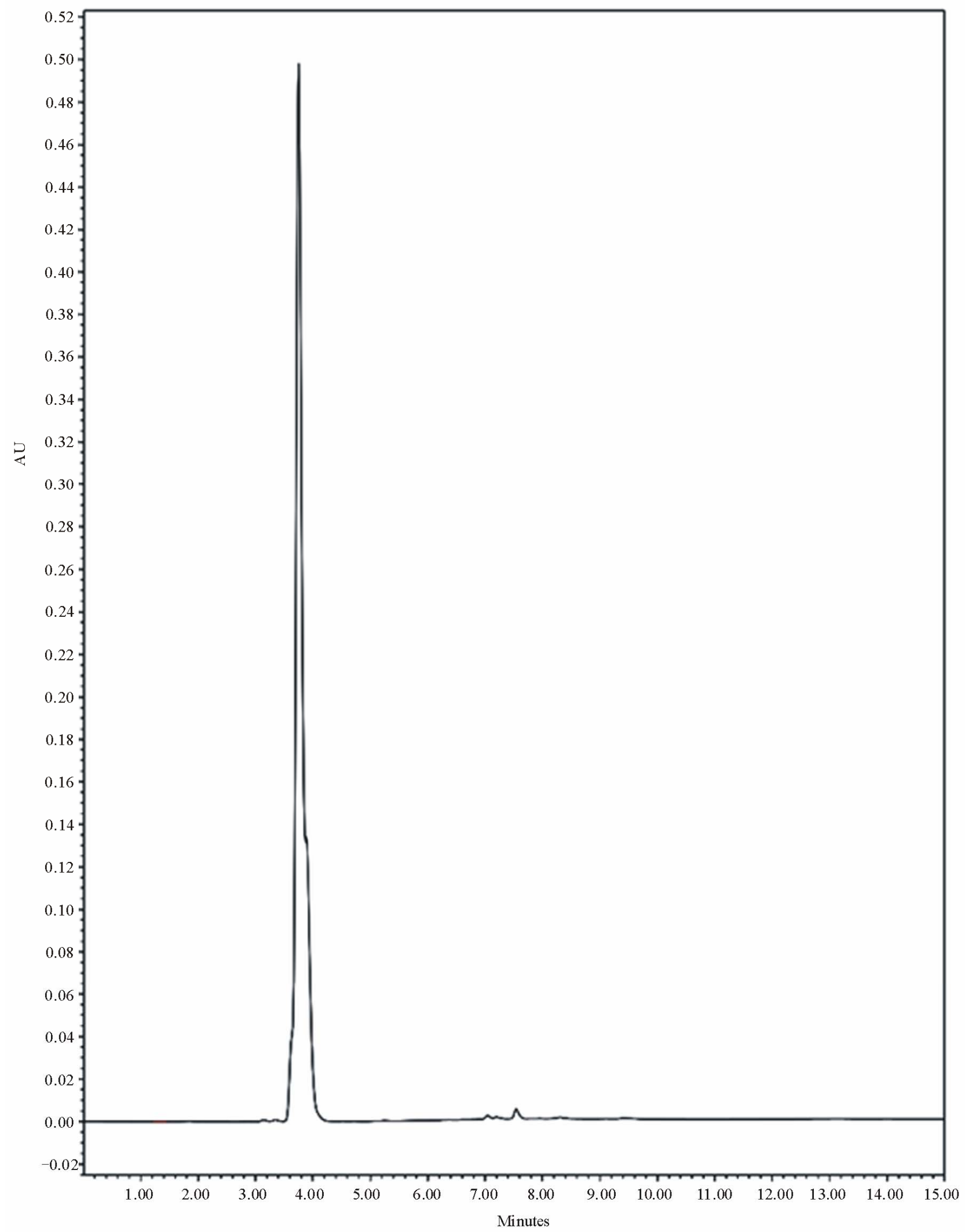

Figure 6. Standard chromatogram's of putrescence extracted with chloroform but separated with methanol or acetonitrile. 


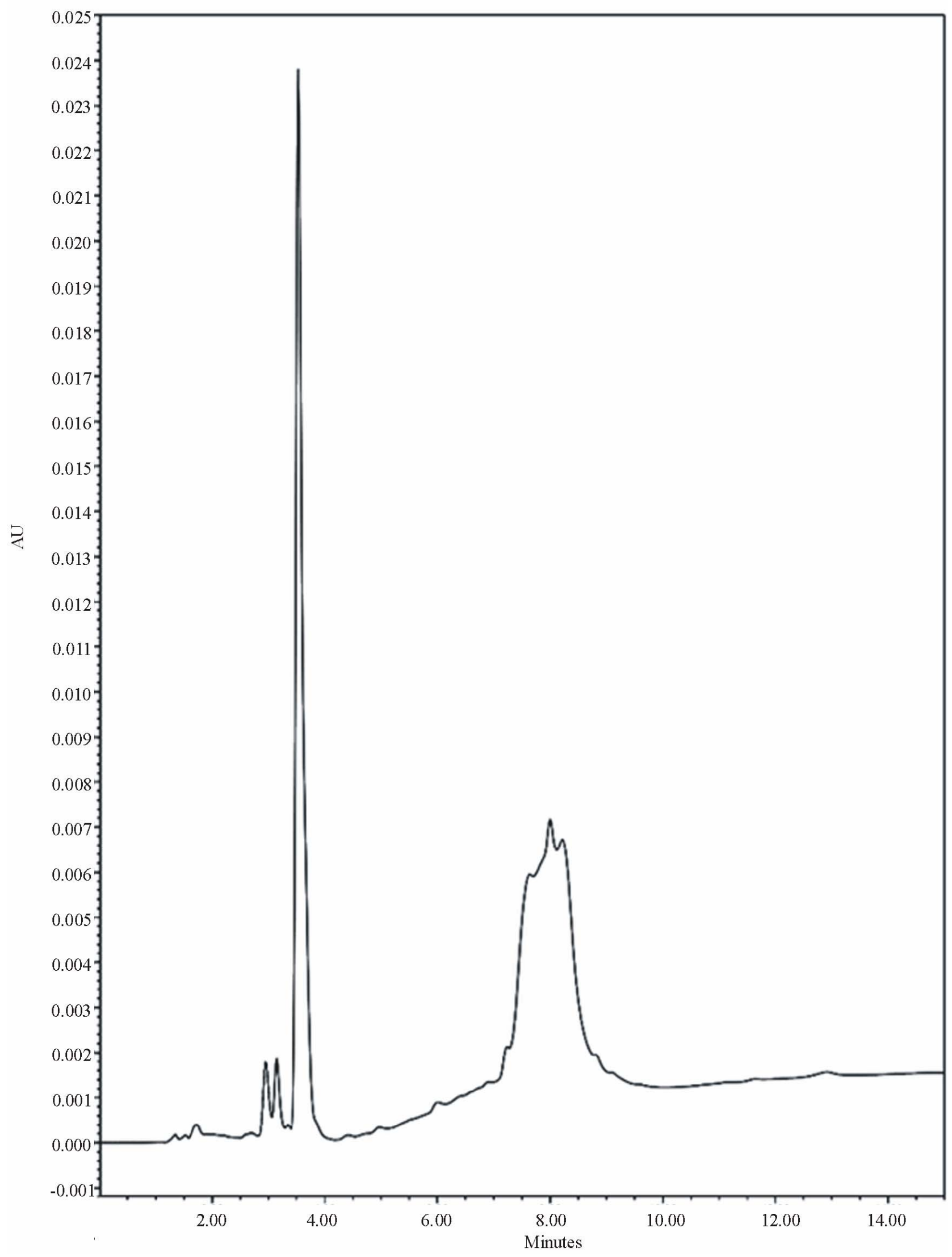




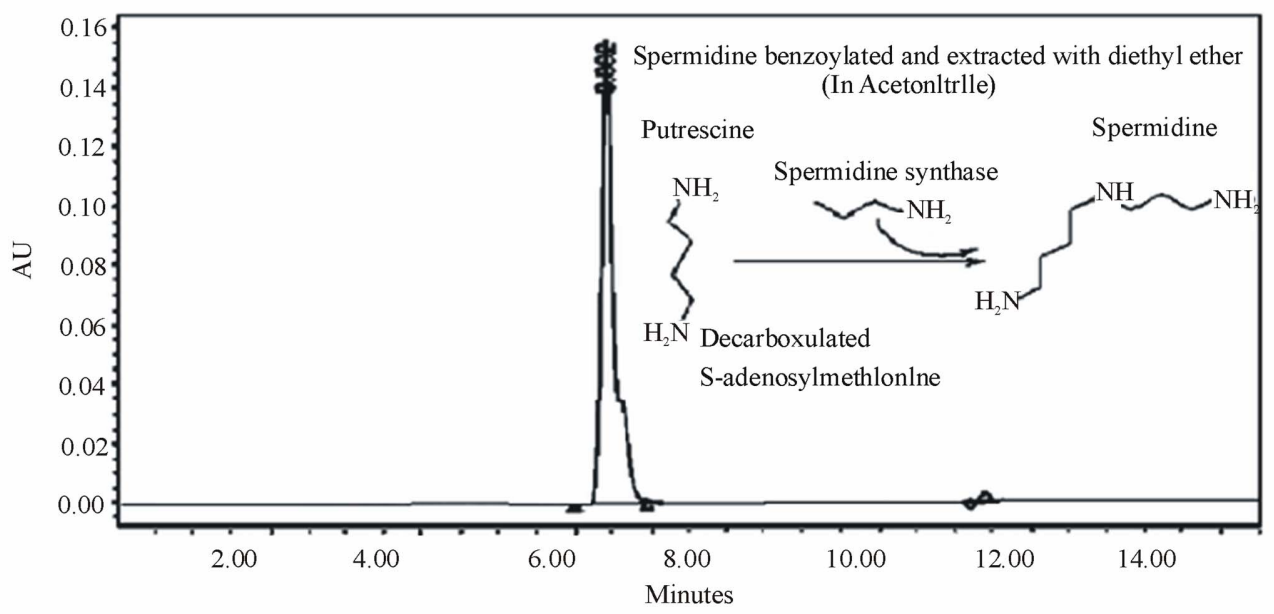

Figure 7. Standard chromatogram's of spermidine extracted with diethyl ether but separated with methanol or acetonitrile.

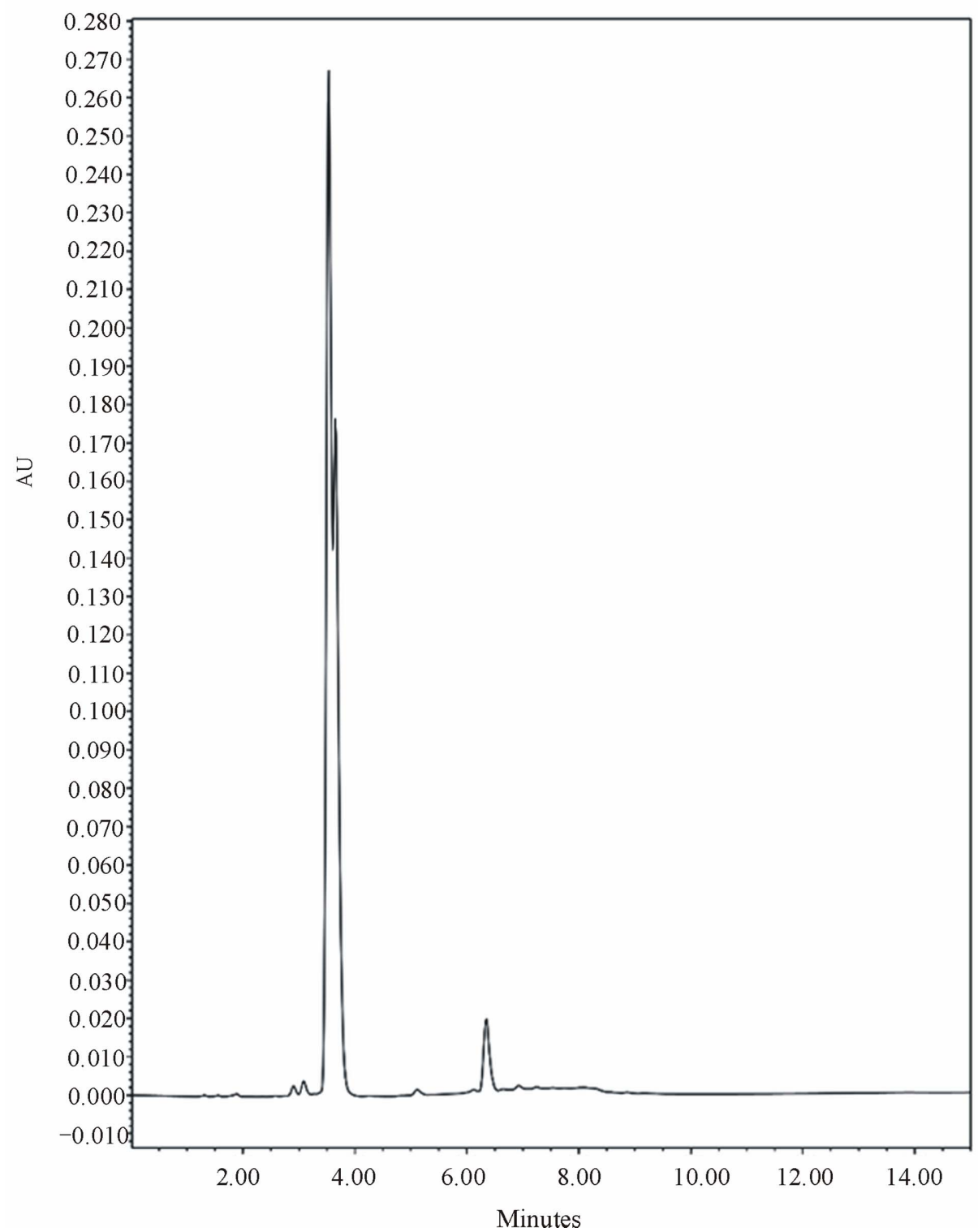




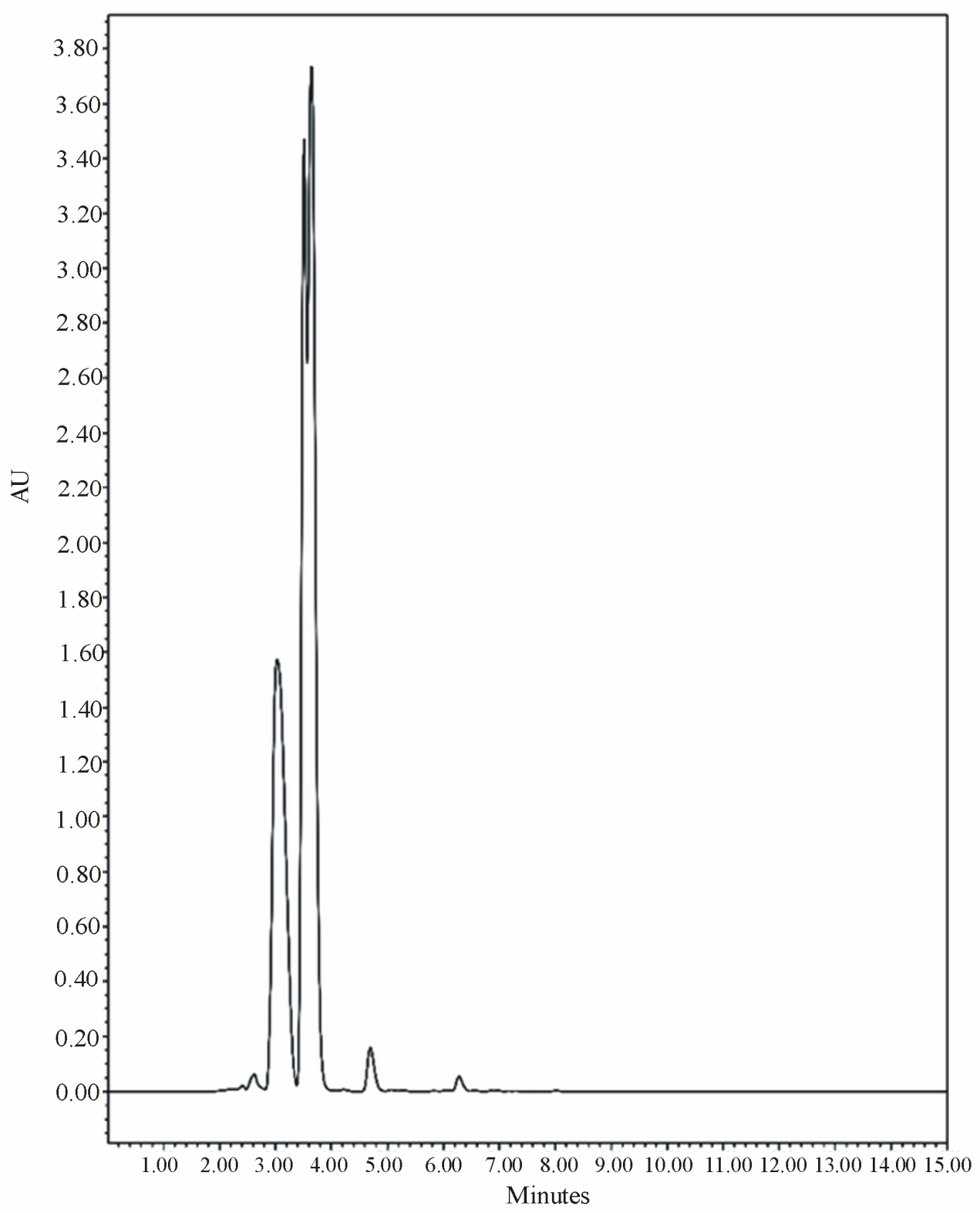

Figure 8. Standard chromatogram's of spermidine extracted with chloroform but separated with methanol or acetonitrile.

Table 3. Relative Recovery and Coefficient of variation from nine standards.

\begin{tabular}{cccc}
\hline Compound & Intra day Mean CV \% & Inter day Mean CV \% & \% Recovery \\
\hline Putrescine & 2.4 & 3.0 & 88.15 \\
Cadaverine & 1.9 & 3.4 & 102.18 \\
Spermidine & 3.0 & 3.8 & 125.25 \\
\hline
\end{tabular}

with acetonitrile was comparable to seperations achieved for putrescine and spermidine in giving very good resolution, accurately and reproducibility for all extracted polyamines.

\section{Discussions}

Accurate analysis of polyamine is important in understanding pathophysiology in tissue stress. And essential 
in understanding disease epidemiology and homeostasis. Our method was able to recover $88 \%$ to $125 \%$ of spiked polyamines derivatized with benzoyl chloride using 1 , 6-diamino hexane as internal standard. Similar trends were observed in the recovery of the internal standard (1,6-diamino hexane). The limit of detection with acetonitrile as solvent was 500 pmoles, which is similar or superior to other published methods which utilize chromatography. The present study, demonstrates greater sensitivity, resolution and reproducibility with the shorter run time to comparable chromatographic-based methods such as pioneered by Morgan [30]. Other advantages were lower consumption of HPLC elution solvent driving down cost of analysis.

\section{Conclusions}

In short, we have demonstrated a modified method based on Morgan's protocol which yielded excellent sensitivity, resolution and a limit of detection of 500 pmoles which is superior to other reported values of 800 pmoles. This method is also more economical by virtue of its lower run time and less consumption of solvents.

\section{Author Contribution}

S. R. Chava undertook all of the experimental work (ex$\operatorname{tr} \equiv$, triplicate analysis) including co-writing of the firme draft of the manuscript. S. Bashir assisted in the extraction and the first set of analyses. He also wrote the first and co-wrote the second and submission draft of the manuscript. M. Castro and R. Sethi co-supervised the student towards his Master of Science thesis, and associated publication and research costs respectively.

\section{Acknowledgements}

We are grateful to Mohammad T. Nutan (TAMHSC) for giving permission to use the HPLC and Mr. Don Marek from the Department of Environmental Engineering (TAMUK) for assistance with the glove box.

\section{References}

[1] L. J. Ignarro, M. L. Balestrieri and C. Napoli, "Nutrition, Physical Activity, and Cardiovascular Disease: an update,” Cardiovascular Research, Vol. 73, No. 2, January 2007, pp. 326-340. doi:10.1016/j.cardiores.2006.06.030

[2] M. L. Daviglus, J. Stamler, A. J. Orencia, A. R. Dyer, K. Liu, P. Greenland, M. K. Walsh, D. Morris and R. B. Shekelle, "Fish Consumption and the 30-Year Risk of Fatal Myocardial Infarction,” The New England Journal of Medicine, Vol. 336, No. 15, April 1997, pp. 1046-1053. doi:10.1056/NEJM199704103361502

[3] A. H. Lichtenstein, L. J. Appel, M. Brands, M. Carnethon,
S. Daniels, H. A. Franch, B. Franklin, P. Kris-Etherton, W. S. Harris, B. Howard, N. Karanja, M. Lefevre, L. Rudel, F. Sacks, L. Van Horn, M. Winston and J. Wylie- Rosett, "Summary of American Heart Association Diet and Lifestyle Recommendations revision 2006,” Arteriosclerosis, Thrombosis, and Vascular Biology, Vol. 26, No. 10, October 2006, pp. 2186-2191.

doi:10.1161/01.ATV.0000238352.25222.5e

[4] Y. J. Zhao, C. Q. Xu, W. H. Zhang, L. Zhang, S. L. Bian, Q. Hang, H. L. Sun, Q. F. Li, Y. Q. Zhang, Y. Tian, R. Wang, B. F. Yang and W. M. Li, "Role of Polyamines in Myocardial Ischemia/Reperfusion Injury and Their Interactions with Nitric Oxide,” European Journal of Pharmacology, Vol. 563, No. 3, May 2007, pp. 236-246. doi:10.1016/j.ejphar.2007.01.096

[5] J. Martin-Tanguy, "Metabolism and Function of Polyamines in Plants: Recent Development (New Approaches),” Plant Growth Regulations, Vol. 34, No. 14, May 2001, pp. 135-148. doi:10.1023/A:1013343106574

[6] M. C. White, R. A. Etzel, W. D. Wilcox and C. Lloyd, "Exacerbations of Childhood Asthma and Ozone Pollution in Atlanta," Environmental Research, Vol. 65, No. 1, April 1994, pp. 56-58. doi:10.1006/enrs.1994.1021

[7] M. D. Denton, H. S. Glazer, D. C. Zellner and F. G. Smith, "Gas-Chromatographic Measurement of Urinary Polyamines in Cancer Patients,” Clinical Chemistry, Vol. 19, No. 8, June 1973, pp. 904-907.

[8] D. R. Roberts, M. A. Walker and E. D. Dumbroff, "Mass Spectral Determination of Benzamide Derivatives of Polyamines Separated by HPLC,” Phytochemistry, Vol. 24, No. 5, January 1995, pp. 1084-1090.

[9] J. W. Redmond and A. Tseng, "High-Pressure Liquid Chromatographic Determination of Putrescine, Cadaverine, Spermidine and Spermine," Journal of Chromatography, Vol. 170, No. 2, March 1979, pp. 479-481. doi:10.1016/S0021-9673(00)95481-5

[10] S. Bardocz, "Effect of Phytohaemagglutinin on Intestinal Cell Proliferation. Role of Polyamines," Archivos Latinoamericanos de Nutrición, Vol. 44, No. 4, Suppl. 1, December 1996, pp. 16S-20S.

[11] A. Gugliucci and T. Menini, "The Polyamines Spermine and Spermidine Protect Proteins from Structural and Functional Damage by AGE Precursors: A New Role for Old Molecules?” Life Science, Vol. 72, No. 23, April 2003, pp. 2603-2616. doi:10.1016/S0024-3205(03)00166-8

[12] S. Wongyai, P. J. Oefner and G. K. Bonn, "High Resolution Analysis of Polyamines and Their Acetyl Derivatives Using RP-HPLC,” Journal of Liquid Chromatography, Vol. 12, No. 12, December 1989, pp. 2249-2261.

[13] G. Taibi and M. R. Schiavo, "Simple High-Performance Liquid Chromatographic Assay for Polyamines and Their Monoacetyl Derivatives,” Journal of Chromatography, Vol. 614, No. 1, April 1993, pp. 153-158.

[14] P. Torrigiani, A. L. Rabiti, L. Betti, F. Marani, M. Bizzi, N. Bagni and A. Canova, "Improved Method for Polyamine Determination in TMV, a Rod-Shaped Virus," 
Journal of Virological Methods, Vol. 53, No. 1, January 1995, pp. 157-163.

[15] J. B. Wehr, "Purification of Plant Polyamines with Anion-Exchange Column Clean-Up Prior to High-Performance Liquid Chromatographic Analysis,” Journal of Chromatography A, Vol. 709, No. 2, August 1995, pp. 241-247. doi:10.1016/0021-9673(95)00461-U

[16] M. Marcé, D. S. Brown, T. Capell, X. Figueras and A. F. Tiburcio, "Rapid High-Performance Liquid Chromatographic Method for the Quantitation of Polyamines as Their Dansyl Derivatives: Application to Plant and Animal Tissues,” Journal of Chromatography B, Vol. 666, No. 2, April 1995, pp. 329-335. doi:10.1016/0378-4347(94)00586-T

[17] H. E. Flores and A. W. Galston, “Analysis of Polyamines in Higher Plants by High Performance Liquid Chromatography,” Plant Physiology, Vol. 69, No. 3, March 1982, pp. 701-706. doi:10.1104/pp.69.3.701

[18] M. A. Smith and P. J. Davies, "Separation and Quantitation of Polyamines in Plant Tissues by High Performance Liquid Chromatography of Their Dansyl Derivatives,” Plant Physiology, Vol. 78, No. 1, May 1985, pp. 89-91. doi:10.1104/pp.78.1.89

[19] R. D. Slocum, H. E. Flores, A. W. Galston and L. H. Weinstein, Improved Method for HPLC Analysis of Polyamines, Agmatine and Aromatic Monoamines in Plant Tissue,” Plant Physiology, Vol. 89, No. 2, February 1989, pp. 512-517. doi:10.1104/pp.89.2.512

[20] P. F. Hockl, S. M. Thyssen and C. Libertun, “An Improved HPLC Method for Identification and Quantitation of Polyamines and Related Compounds as Benzoylated Derivatives," Journal of Liquid Chromatography \& Related Technologies, Vol. 23, No. 5, March 2000, pp. 693-703. doi:10.1081/JLC-100101482

[21] A. Gamarick and R. B. Frydman, "Cadaverine, an Essential Diamine for the Normal Root Development of Germinating Soybean (Glycine max) Seeds," Plant Physiology, Vol. 97, No. 2, February 1991, pp. 778-785. doi:10.1104/pp.97.2.778

[22] R. M. Adibhatla, J. F. Hatcher, K. Sailor and R. J. Dempsey, "Polyamines and Central Nervous System Injury: Spermine and Spermidine Decrease Following Transient Focal Cerebral Ischemia in Spontaneously Hypertensive Rats,” Brain Research, Vol. 938, No. 1-2, May 2002, pp. 81-86. doi:10.1016/S0006-8993(02)02447-2
[23] W. Paschen, R. Widmann and C. Weber, "Changes in Regional Polyamine Profiles in Rat Brains after Transient Cerebral Ischemia (Single versus Repetitive Ischemia): Evidence for Release of Polyamines from Injured Neurons," Neuroscience Letters, Vol. 135, No. 1, January 1992, pp. 121-124. doi:10.1016/0304-3940(92)90150-6

[24] C. N. Ramchand, I. Das, A. Gliddon and S. R. Hirsch, "Role of Polyamines in the Membrane Pathology of Schizophrenia A Study Using Fibroblasts from Schizophrenic Patients and Normal Controls," Schizophrenia Research, Vol. 13, No. 3, October 1994, pp. 249-253. doi:10.1016/0920-9964(94)90049-3

[25] W. Paschen, L. Csiba, G. Rohn and D. Bereczki, "Polyamine Metabolism in Transient Focal Ischemia of Rat Brain,” Brain Research, Vol. 566, No. 1-2, December 1991, pp. 354-357. doi:10.1016/0006-8993(91)91726-H

[26] C. Strambi, A. Tirard, M. Renucci, P. Faure, P. Charpin, R. Augier and A. Strambi, "Ecdysone Deprivation Affects Polyamine Metabolism in the House Cricket Acheta Domesticus," Insect Biochemistry and Molecular Biology, Vol. 23, No. 1, January 1993, pp. 165-170. doi:10.1016/0965-1748(93)90096-B

[27] K. Kotzabasis, M. D. Christakis and K. A. Roubelakis-Angelakis, "A Narrow-Bore HPLC Method for the Identification and Quantitation of Free, Conjugated, and Bound Polyamines,” Analytical Biochemistry, Vol. 214, No. 2, November 1993, pp. 484-489. doi:10.1006/abio.1993.1526

[28] C. Cann-Moissan, J. Caroff, A. Hourmant, C. Videau and F. Rapt, "Quantitative Analysis of Polyamines at Trace Levels by High Performance Liquid Chromatography in High Salt Solutions. Application to Seawater," Journal of Liquid Chromatography, Vol. 17, No. 16, December 1994, pp. 1413-1417. doi:10.1080/10826079408013773

[29] C. F. Verkolen, J. C. Romijn, F. H. Schroeder, W. P. Schalkwijt and T. A. Splinter, "Quantitation of Polyamines in Cultured Cells and Tissue Homogenates by Reversed-Phase High-Performance Liquid Chromatography of Their Benzoyl Derivatives," Journal of Chromatography, Vol. 426, No. 11, November 1988, pp. 4154.

[30] D. M. L. Morgan, "Determination of Polyamines as Their Benzoylated Derivatives by HPLC,” In: D. M. L. Morgan, Ed., Polyamine Protocols, Humana Press, Totowa, 1997, pp. 111-118. doi:10.1385/0-89603-448-8:111 